\title{
A note on double Laplace decomposition method for solving singular one dimensional pseudo thermo-elasticity coupled system
}

\author{
Hassan Eltayeb*, Imed Bachar \\ Mathematics Department, College of Science, King Saud University, P. O. Box 2455, Riyadh 11451, Saudi Arabia. \\ Communicated by $\mathrm{X} .-\mathrm{J}$. Yang
}

\begin{abstract}
In this paper, Adomain decomposition method is reintroduced with double Laplace transform methods to obtain closed form solutions of linear and nonlinear singular one dimensional pseudo thermo-elasticity coupled system. The nonlinear terms can be easily handled by the use of Adomian polynomials. Furthermore, we illustrate our proposed methods by one example.
\end{abstract}

Keywords: Double Laplace transform, inverse Laplace transform, pseudo thermo-elasticity equation, single Laplace transform, decomposition methods.

2010 MSC: 35A22, 44A10.

(C)2018 All rights reserved.

\section{Introduction}

The theory of generalized thermo-elasticity with one relaxation time for an isotropic body was introduced by Lord and Shulman [17]. This theory corrects the unrealistic conclusions of the previous theories (the uncoupled and the coupled theories of thermo-elasticity) which tells that heat waves travel with infinite speeds. The one dimensional thermo-elasticity coupled system was one of the first domains in coupled field theory that attracted the mathematicians. The thermo-elasticity problems occur in different fields of engineering, physics, and biology. Many authors have been studied, see [12, 23] are applied homotopy perturbation and variational iteration methods for the solution of nonlinear thermo-elasticity coupled systems. Recently, many methods have been used for solution of linear and nonlinear problem, for example, Adomian decomposition method (ADM); see [4, 8, 9, 21, 22] and iteration method [5, 6]. In [20]. The authors have solved a particular case of the given nonlinear problem by combining a functional analysis and iteration method. Many authors has been studied the convergence analysis of Adomian's method; see $[1-3,7,11]$. The aim of this paper is to solve linear and nonlinear singular one dimensional

\footnotetext{
${ }^{*}$ Corresponding author

Email addresses: hgadain@ksu.edu.sa (Hassan Eltayeb), abachar@ksu.edu.sa (Imed Bachar)

doi: $10.22436 /$ jnsa.011.06.12
}

Received: 2017-06-04 Revised: 2018-01-10 Accepted: 2018-01-12 
pseudo thermo-elasticity coupled system by using the combine domain decomposition techniques and double Laplace transform methods and to study the sufficient condition of convergence of our methods. Now, we recall the following definitions which are given by $[10,15,16]$. The double Laplace transform is defined as

$$
\mathrm{L}_{\mathrm{x}} \mathrm{L}_{\mathrm{t}}[\mathrm{f}(\mathrm{x}, \mathrm{t})]=\mathrm{F}(\mathrm{p}, \mathrm{s})=\int_{0}^{\infty} \int_{0}^{\infty} e^{-\mathrm{px}-\mathrm{st}} \mathrm{f}(\mathrm{x}, \mathrm{t}) \mathrm{dt} \mathrm{d} \mathrm{x},
$$

where $x, t>0$ and $p, s$ are complex values, and further double Laplace transform of the first order partial derivatives is given by

$$
\mathrm{L}_{x} \mathrm{~L}_{\mathrm{t}}\left[\frac{\partial \mathrm{f}(\mathrm{x}, \mathrm{t})}{\partial x}\right]=p \mathrm{~F}(\mathrm{p}, \mathrm{s})-\mathrm{F}(0, \mathrm{~s}) .
$$

Similarly the double Laplace transform for second partial derivative with respect to $x$ and $t$ are defined as follows

$$
\begin{aligned}
& L_{x} L_{t}\left[\frac{\partial^{2} u(x, t)}{\partial^{2} x}\right]=p^{2} u(p, s)-p u(0, s)-\frac{\partial u(0, s)}{\partial x}, \\
& L_{x} L_{t}\left[\frac{\partial^{2} u(x, t)}{\partial^{2} t}\right]=s^{2} u(p, s)-s u(p, 0)-\frac{\partial u(p, 0)}{\partial t} .
\end{aligned}
$$

The following are basic definitions of the double Laplace transform, which shall be used in this paper.

Definition 1.1. The double Laplace transform of the functions $x \frac{\partial v}{\partial t}, x \frac{\partial^{2} u}{\partial t^{2}}, x f(x, t)$ and $x g(x, t)$ are given by

$$
\begin{aligned}
\mathrm{L}_{\chi} \mathrm{L}_{t}\left(\chi \frac{\partial v}{\partial t}\right) & =-\frac{d}{d p}[s V(p, s)-V(p, 0)] \\
\mathrm{L}_{x} \mathrm{~L}_{t}\left(\chi \frac{\partial^{2} u}{\partial \mathrm{t}^{2}}\right) & =-\frac{d}{d p}\left[s^{2} \mathrm{u}(p, s)-s u(p, 0)-\frac{\partial u(p, 0)}{\partial t}\right]
\end{aligned}
$$

and

$$
\begin{aligned}
& L_{x} L_{t}(x f(x, t))=-\frac{d}{d p}\left[L_{x} L_{t}(f(x, t))\right]=-\frac{d F(p, s)}{d p} \\
& L_{x} L_{t}(x g(x, t))=-\frac{d}{d p}\left[L_{x} L_{t}(g(x, t))\right]=-\frac{d G(p, s)}{d p} .
\end{aligned}
$$

\section{Linear singular one dimensional pseudo thermo-elasticity coupled system}

In this section, we apply the double Laplace Adomian decomposition method to solve the linear singular one dimensional pseudo thermo-elasticity coupled system given by

$$
\begin{array}{cc}
\frac{\partial^{2} u}{\partial t^{2}}-\frac{a}{x} \frac{\partial}{\partial x}\left(x \frac{\partial u}{\partial x}\right)-\frac{b \phi(t)}{x} \frac{\partial^{2}}{\partial x \partial t}\left(x \frac{\partial u}{\partial x}\right)+c \psi(x) \frac{\partial v}{\partial x}=f(x, t), & x \in \Omega, \\
\frac{\partial v}{\partial t}-\frac{d}{x} \frac{\partial}{\partial x}\left(x \frac{\partial v}{\partial x}\right)-\frac{e \varphi(t)}{x} \frac{\partial^{2}}{\partial x \partial t}\left(x \frac{\partial v}{\partial x}\right)+m \delta(x, t) \frac{\partial^{2} u}{\partial x \partial t}=g(x, t), & t>0,
\end{array}
$$

subject to

$$
u(x, 0)=f_{1}(x), \frac{\partial u(x, 0)}{\partial t}=f_{2}(x), \quad v(x, 0)=g_{1}(x)
$$

where

$$
\frac{\mathrm{a}}{x} \frac{\partial}{\partial x}\left(x \frac{\partial u}{\partial x}\right), \frac{\mathrm{d}}{x} \frac{\partial}{\partial x}\left(x \frac{\partial v}{\partial x}\right)
$$


are called Bessel's operators, $a, b, c, d, e$, and $m$ are constants and $f(x, t), g(x, t), \delta(x, t), \phi(t), \varphi(t)$, and $\psi(x)$ are known functions. To obtain the solution of Linear singular one dimensional pseudo thermoelasticity coupled system of (2.1), first we multiply both sides of (2.1) by $x$, and using the definition of partial derivatives of the double Laplace transform and single Laplace transform for (2.1) and (2.2) respectively and Definition 1.1, we get

$$
\frac{d U(p, s)}{d p}=\frac{d F_{1}(p)}{s d p}+\frac{d F_{2}(p)}{s^{2} d p}+\frac{d F(p, s)}{s^{2} d p}-\frac{1}{s^{2}} L_{x} L_{t}\left[\Psi-c x \psi(x) \frac{\partial v}{\partial x}\right]
$$

and

$$
\frac{d V(p, s)}{d p}=\frac{d G_{1}(p)}{s d p}+\frac{d G(p, s)}{s d p}-\frac{1}{s} L_{x} L_{t}\left[\Phi-m x \delta(x, t) \frac{\partial^{2} u}{\partial x \partial t}\right]
$$

where

$$
\Psi=a \frac{\partial}{\partial x}\left(x \frac{\partial u}{\partial x}\right)+b \phi(t) \frac{\partial^{2}}{\partial x \partial t}\left(x \frac{\partial u}{\partial x}\right), \Phi=d \frac{\partial}{\partial x}\left(x \frac{\partial v}{\partial x}\right)+e \varphi(t) \frac{\partial^{2}}{\partial x \partial t}\left(x \frac{\partial v}{\partial x}\right),
$$

and $F_{1}(p), F_{2}(p)$, and $G_{1}(p)$ are single Laplace transforms of $f_{1}(x), f_{2}(x)$, and $g_{1}(x)$, respectively. By integrating both sides of (2.3) and (2.4) from 0 to $p$ with respect to $p$, we get

$$
U(p, s)=\int_{0}^{p}\left(\frac{d F_{1}(p)}{s d p}+\frac{d F_{1}(p)}{s^{2} d p}+\frac{d F(p, s)}{s^{2} d p}\right) d p-\frac{1}{s^{2}} \int_{0}^{p}\left(L_{x} L_{t}\left[\Psi-c x \psi(x) \frac{\partial v}{\partial x}\right]\right) d p
$$

and

$$
V(p, s)=\int_{0}^{p}\left(\frac{d G_{1}(p)}{s d p}+\frac{d G(p, s)}{s d p}\right) d p-\frac{1}{s} \int_{0}^{p} L_{x} L_{t}\left[\Phi-m x \delta(x, t) \frac{\partial^{2} u}{\partial x \partial t}\right] d p .
$$

The double Laplace a domain decomposition methods (DLADM) defines the solution of the system as $u(x, t)$ and $v(x, t)$ by the infinite series

$$
u(x, t)=\sum_{n=0}^{\infty} u_{n}(x, t), v(x, t)=\sum_{n=0}^{\infty} v_{n}(x, t)
$$

By applying double inverse Laplace transform for (2.6) and (2.7), we have

$$
u(x, t)=L_{p}^{-1} L_{s}^{-1}\left[\int_{0}^{p}\left(\frac{d F_{1}(p)}{s d p}+\frac{d F_{1}(p)}{s^{2} d p}+\frac{d F(p, s)}{s^{2} d p}\right) d p\right]-L_{p}^{-1} L_{s}^{-1}\left[\frac{1}{s^{2}} \int_{0}^{p}\left(L_{x} L_{t}\left[\Psi-c x \psi(x) \frac{\partial v}{\partial x}\right]\right) d p\right]
$$

and

$$
v(x, t)=L_{p}^{-1} L_{s}^{-1}\left[\int_{0}^{p}\left(\frac{d G_{1}(p)}{s d p}+\frac{d G(p, s)}{s d p}\right) d p\right]+L_{p}^{-1} L_{s}^{-1}\left[\frac{1}{s} \int_{0}^{p} L_{x} L_{t}\left[\Phi-m x \delta(x, t) \frac{\partial^{2} u}{\partial x \partial t}\right] d p\right],
$$

therefore

$$
\begin{aligned}
u(x, t)= & f_{1}(x)+t f_{2}(x)+L_{p}^{-1} L_{s}^{-1}\left[\frac{1}{s^{2}} \int_{0}^{p} d F(p, s)\right] \\
& -L_{p}^{-1} L_{s}^{-1}\left[\frac{1}{s^{2}} \int_{0}^{p}\left(L_{x} L_{t}\left[\Psi-c x \psi(x) \frac{\partial v}{\partial x}\right]\right) d p\right]
\end{aligned}
$$

and

$$
v(x, t)=g_{1}(x)+L_{p}^{-1} L_{s}^{-1}\left[\frac{1}{s} \int_{0}^{p} d G(p, s)\right]-L_{p}^{-1} L_{s}^{-1}\left[\frac{1}{s} \int_{0}^{p} L_{x} L_{t}\left[\Phi-m x \delta(x, t) \frac{\partial^{2} u}{\partial x \partial t}\right] d p\right] .
$$


Substituting (2.8) into (2.9) and (2.10), we get

$$
\begin{aligned}
\sum_{n=0}^{\infty} u_{n}(x, t)= & f_{1}(x)+t f_{2}(x)+L_{p}^{-1} L_{s}^{-1}\left[\frac{1}{s^{2}} \int_{0}^{p} d F(p, s)\right]-L_{p}^{-1} L_{s}^{-1}\left[\frac{1}{s^{2}} \int_{0}^{p} L_{x} L_{t}\left[\sum_{n=0}^{\infty} \Psi_{n}\right] d p\right] \\
& +L_{p}^{-1} L_{s}^{-1}\left[\frac{1}{s^{2}} \int_{0}^{p} L_{x} L_{t}\left[c x \psi(x) \sum_{n=0}^{\infty} v_{n x}(x, t)\right] d p\right]
\end{aligned}
$$

and

$$
\begin{aligned}
\sum_{n=0}^{\infty} v_{n}(x, t)= & g_{1}(x)+L_{p}^{-1} L_{s}^{-1}\left[\frac{1}{s} \int_{0}^{p} d G(p, s)\right]-L_{p}^{-1} L_{s}^{-1}\left[\frac{1}{s} \int_{0}^{p}\left(L_{x} L_{t}\left[\sum_{n=0}^{\infty} \Phi_{n}\right]\right) d p\right] \\
& +L_{p}^{-1} L_{s}^{-1}\left[\frac{1}{s} \int_{0}^{p}\left(L_{x} L_{t}\left[m x \delta(x, t) \frac{\partial^{2}}{\partial x \partial t}\left(\sum_{n=0}^{\infty} u_{n}(x, t)\right)\right]\right) d p\right]
\end{aligned}
$$

The decomposition method suggests that the zeroth components $u_{0}$ and $v_{0}$ are identified by the terms arising from the initial conditions and from source terms as shown

$$
\begin{aligned}
& u_{0}(x, t)=f_{1}(x)+t f_{2}(x)+L_{p}^{-1} L_{s}^{-1}\left[\frac{1}{s^{2}} \int_{0}^{p} d F(p, s)\right], \\
& v_{0}(x, t)=g_{1}(x)+L_{p}^{-1} L_{s}^{-1}\left[\frac{1}{s} \int_{0}^{p} d G(p, s)\right],
\end{aligned}
$$

for more details see [24]. The remaining components of $u(x, t)$ and $v(x, t)$ are determined in a recursive manner from (2.11), (2.12), and using (2.5) as follows

$$
\begin{aligned}
& u_{1}(x, t)=-L_{p}^{-1} L_{s}^{-1}\left[\frac{1}{s^{2}} \int_{0}^{p} L_{x} L_{t}\left[a \frac{\partial}{\partial x}\left(x \frac{\partial u_{0}}{\partial x}\right)\right] d p\right] \\
& -\mathrm{L}_{\mathrm{p}}^{-1} \mathrm{~L}_{\mathrm{s}}^{-1}\left[\frac{1}{\mathrm{~s}^{2}} \int_{0}^{p} \mathrm{~L}_{\chi} \mathrm{L}_{\mathrm{t}}\left[\mathrm{b} \phi(\mathrm{t}) \frac{\partial^{2}}{\partial x \partial t}\left(x \frac{\partial \mathrm{u}_{0}}{\partial x}\right)\right] \mathrm{dp}\right] \\
& +\mathrm{L}_{\mathrm{p}}^{-1} \mathrm{~L}_{\mathrm{s}}^{-1}\left[\frac{1}{\mathrm{~s}^{2}} \int_{0}^{\mathrm{p}} \mathrm{L}_{\chi} \mathrm{L}_{\mathrm{t}}\left[\mathrm{c} \chi \psi(x) \frac{\partial v_{0}}{\partial x}\right] \mathrm{dp}\right] \text {, } \\
& v_{1}(x, t)=-L_{p}^{-1} L_{s}^{-1}\left[\frac{1}{s} \int_{0}^{p}\left(L_{x} L_{t}\left[d \frac{\partial}{\partial x}\left(x \frac{\partial v_{0}}{\partial x}\right)\right]\right) d p\right] \\
& -\mathrm{L}_{p}^{-1} \mathrm{~L}_{s}^{-1}\left[\frac{1}{s} \int_{0}^{p}\left(\mathrm{~L}_{x} \mathrm{~L}_{\mathrm{t}}\left[e \varphi(t) \frac{\partial^{2}}{\partial x \partial t}\left(x \frac{\partial v_{0}}{\partial x}\right)\right]\right) d p\right] \\
& +\mathrm{L}_{\mathrm{p}}^{-1} \mathrm{~L}_{\mathrm{s}}^{-1}\left[\frac{1}{\mathrm{~s}} \int_{0}^{\mathrm{p}}\left(\mathrm{L}_{x} \mathrm{~L}_{\mathrm{t}}\left[\mathrm{m} x \delta(x, \mathrm{t}) \frac{\partial^{2} \mathrm{u}_{0}}{\partial x \partial \mathrm{t}}\right]\right) \mathrm{dp}\right],
\end{aligned}
$$

and

$$
\begin{aligned}
u_{2}(x, t)= & -L_{p}^{-1} L_{s}^{-1}\left[\frac{1}{s^{2}} \int_{0}^{p} L_{x} L_{t}\left[a \frac{\partial}{\partial x}\left(x \frac{\partial u_{1}}{\partial x}\right)\right] d p\right] \\
& -L_{p}^{-1} L_{s}^{-1}\left[\frac{1}{s^{2}} \int_{0}^{p} L_{x} L_{t}\left[b \phi(t) \frac{\partial^{2}}{\partial x \partial t}\left(x \frac{\partial u_{1}}{\partial x}\right)\right] d p\right] \\
& +L_{p}^{-1} L_{s}^{-1}\left[\frac{1}{s^{2}} \int_{0}^{p} L_{x} L_{t}\left[c x \psi(x) \frac{\partial v_{1}}{\partial x}\right] d p\right] \\
v_{2}(x, t)= & -L_{p}^{-1} L_{s}^{-1}\left[\frac{1}{s} \int_{0}^{p}\left(L_{x} L_{t}\left[d \frac{\partial}{\partial x}\left(x \frac{\partial v_{1}}{\partial x}\right)\right]\right) d p\right]
\end{aligned}
$$




$$
\begin{aligned}
& -\mathrm{L}_{p}^{-1} \mathrm{~L}_{s}^{-1}\left[\frac{1}{s} \int_{0}^{p}\left(\mathrm{~L}_{x} \mathrm{~L}_{\mathrm{t}}\left[e \varphi(t) \frac{\partial^{2}}{\partial x \partial t}\left(x \frac{\partial v_{1}}{\partial x}\right)\right]\right) d p\right] \\
& +\mathrm{L}_{p}^{-1} \mathrm{~L}_{s}^{-1}\left[\frac{1}{s} \int_{0}^{p}\left(\mathrm{~L}_{x} \mathrm{~L}_{\mathrm{t}}\left[m x \delta(x, t) \frac{\partial^{2} \mathrm{u}_{1}}{\partial x \partial t}\right]\right) d p\right] .
\end{aligned}
$$

In general, the recursive relation is given by

$$
\begin{aligned}
u_{n+1}(x, t)= & -L_{p}^{-1} L_{s}^{-1}\left[\frac{1}{s^{2}} \int_{0}^{p} L_{x} L_{t}\left[a\left(x \sum_{n=0}^{\infty} u_{n x}(x, t)\right)_{x}\right] d p\right] \\
& -L_{p}^{-1} L_{s}^{-1}\left[\frac{1}{s^{2}} \int_{0}^{p} L_{x} L_{t}\left[b \phi(t)\left(x \sum_{n=0}^{\infty} u_{n x}(x, t)\right)_{x t}\right] d p\right] \\
& +L_{p}^{-1} L_{s}^{-1}\left[\frac{1}{s^{2}} \int_{0}^{p} L_{x} L_{t}\left[c x \psi(x) \sum_{n=0}^{\infty} v_{n x}(x, t)\right] d p\right]
\end{aligned}
$$

and

$$
\begin{aligned}
v_{n+1}(x, t)= & -L_{p}^{-1} L_{s}^{-1}\left[\frac{1}{s} \int_{0}^{p}\left(L_{x} L_{t}\left[d\left(\sum_{n=0}^{\infty} v_{n x}(x, t)\right)_{x}\right]\right) d p\right] \\
& -L_{p}^{-1} L_{s}^{-1}\left[\frac{1}{s} \int_{0}^{p}\left(L_{x} L_{t}\left[e \varphi(t)\left(\sum_{n=0}^{\infty} v_{n x}(x, t)\right)_{x t}\right]\right) d p\right] \\
& +L_{p}^{-1} L_{s}^{-1}\left[\frac{1}{s} \int_{0}^{p}\left(L_{x} L_{t}\left[m x \delta(x, t)\left(\sum_{n=0}^{\infty} u_{n}(x, t)\right)_{x t}\right]\right) d p\right] .
\end{aligned}
$$

Here, we assume that the double inverse Laplace transform exists for each term in the right hand side of (2.13), (2.14), and (2.15). Hence, to obtain the solution of the system we calculate the terms $u_{0}, u_{1}, \ldots$ and $v_{0}, v_{1}, \ldots$

In the following example, we consider $a=b=e=d=1, c=m=4$, and $\varphi=, \phi=t, \psi(x)=$ $\frac{1}{x}, \delta(x, t)=\frac{t}{x}, f(x, t)=g(x, t)=0$, in (2.1).

Example 2.1. Consider the following linear singular one dimensional thermo-elasticity coupled system

$$
\begin{gathered}
\frac{\partial^{2} u}{\partial t^{2}}-\frac{1}{x}\left(x \frac{\partial u}{\partial x}\right)_{x}-\frac{t}{x} \frac{\partial^{2}}{\partial x \partial t}\left(x \frac{\partial u}{\partial x}\right)+\frac{4}{x} \frac{\partial v}{\partial x}=0, \\
\frac{\partial v}{\partial t}-\frac{1}{x}\left(x \frac{\partial v}{\partial x}\right)_{x}-\frac{t}{x} \frac{\partial^{2}}{\partial x \partial t}\left(x \frac{\partial v}{\partial x}\right)+\frac{4 t}{x} \frac{\partial^{2} u}{\partial x \partial t}=x^{2},
\end{gathered}
$$

subject to

$$
u(x, 0)=0, \frac{\partial u(x, 0)}{\partial t}=x^{2}, \quad v(x, 0)=0 .
$$

First multiplying both sides of (2.16) by $x$, using the differentiation property of double Laplace transform and single Laplace transform for (2.16) and (2.17), respectively and Definition 1.1 we obtain

$$
\frac{d U(p, s)}{d p}=-\frac{6}{p^{4} s^{2}}-\frac{1}{s^{2}} L_{x} L_{t}\left[\frac{\partial}{\partial x}\left(x \frac{\partial u}{\partial x}\right)+t \frac{\partial^{2}}{\partial x \partial t}\left(x \frac{\partial u}{\partial x}\right)-4 \frac{\partial v}{\partial x}\right]
$$

and

$$
\frac{d V(p, s)}{d p}=-\frac{6}{p^{4} s^{2}}-\frac{1}{s} L_{x} L_{t}\left[\frac{\partial}{\partial x}\left(x \frac{\partial v}{\partial x}\right)+t \frac{\partial^{2}}{\partial x \partial t}\left(x \frac{\partial v}{\partial x}\right)-4 t \frac{\partial^{2} u}{\partial x \partial t}\right]
$$


Second, by integrating both sides of (2.18) and (2.19) from 0 to $p$ with respect to $p$, applying the inverse double Laplace transform, and using (2.8), we have

$$
\begin{aligned}
u= & x^{2} t-L_{p}^{-1} L_{s}^{-1}\left[\frac{1}{s^{2}} \int_{0}^{p} L_{x} L_{t}\left[\left(x \sum_{n=0}^{\infty} u_{n x}(x, t)\right)_{x}\right] d p\right] \\
& -L_{p}^{-1} L_{s}^{-1}\left[\frac{1}{s^{2}} \int_{0}^{p} L_{x} L_{t}\left[t\left(x \sum_{n=0}^{\infty} u_{n x}(x, t)\right)_{x t}\right] d p\right] \\
& +L_{p}^{-1} L_{s}^{-1}\left[\frac{1}{s^{2}} \int_{0}^{p} L_{x} L_{t}\left[4 \sum_{n=0}^{\infty} v_{n x}(x, t)\right] d p\right]
\end{aligned}
$$

and

$$
\begin{aligned}
& v=x^{2} \mathrm{t}-\mathrm{L}_{\mathrm{p}}^{-1} \mathrm{~L}_{\mathrm{s}}^{-1}\left[\frac{1}{\mathrm{~s}} \int_{0}^{\mathrm{p}}\left(\mathrm{L}_{\chi} \mathrm{L}_{\mathrm{t}}\left[\left(x \sum_{n=0}^{\infty} v_{n x}(x, \mathrm{t})\right)_{\chi}\right]\right) \mathrm{dp}\right] \\
& -\mathrm{L}_{\mathrm{p}}^{-1} \mathrm{~L}_{\mathrm{s}}^{-1}\left[\frac{1}{\mathrm{~s}} \int_{0}^{\mathrm{p}}\left(\mathrm{L}_{\mathrm{x}} \mathrm{L}_{\mathrm{t}}\left[\mathrm{t}\left(x \sum_{\mathrm{n}=0}^{\infty} v_{\mathrm{n} x}(x, \mathrm{t})\right)_{\mathrm{x}}\right]\right) \mathrm{dp}\right] \\
& +\mathrm{L}_{\mathrm{p}}^{-1} \mathrm{~L}_{\mathrm{s}}^{-1}\left[\frac{1}{\mathrm{~s}} \int_{0}^{p}\left(\mathrm{~L}_{\mathrm{x}} \mathrm{L}_{\mathrm{t}}\left[4 \mathrm{t} \frac{\partial^{2}}{\partial x \partial t}\left(\sum_{n=0}^{\infty} \mathrm{u}_{\mathrm{n}}(\mathrm{x}, \mathrm{t})\right)\right]\right) \mathrm{dp}\right] \text {. }
\end{aligned}
$$

On using (2.13), (2.14), and (2.15), we get

$$
\mathrm{u}_{0}=x^{2} \mathrm{t}, \quad v_{0}=x^{2} \mathrm{t}
$$

and

$$
\begin{aligned}
u_{1} & =-L_{p}^{-1} L_{s}^{-1}\left[\frac{1}{s^{2}} \int_{0}^{p}\left(L_{x} L_{t}\left[\left(x u_{0 x}\right)_{x}+t\left(x u_{0 x}\right)_{x t}-4 v_{0 x}\right]\right) d p\right] \\
& =-L_{p}^{-1} L_{s}^{-1}\left[\frac{1}{s^{2}} \int_{0}^{p}\left(L_{x} L_{t}[4 x t+4 x t-8 x t]\right) d p\right], \\
v_{1} & =-L_{p}^{-1} L_{s}^{-1}\left[\frac{1}{s} \int_{0}^{p}\left(L_{x} L_{t}\left[\left(x v_{0 x}\right)_{x}+t\left(x v_{0 x}\right)_{x t}-x^{2} u_{0 x t}\right]\right) d p\right] \\
& =-L_{p}^{-1} L_{s}^{-1}\left[\frac{1}{s} \int_{0}^{p}\left(L_{x} L_{t}[4 x t+4 x t-8 x t]\right) d p\right], \\
u_{1} & =0, v_{1}=0,
\end{aligned}
$$

and

$$
\begin{aligned}
& u_{2}=-\mathrm{L}_{p}^{-1} \mathrm{~L}_{s}^{-1}\left[\frac{1}{s^{2}} \int_{0}^{p}\left(\mathrm{~L}_{x} \mathrm{~L}_{\mathrm{t}}\left[\left(x \mathrm{u}_{1 x}\right)_{x}+\left(x u_{1 x}\right)_{x t}-x^{2} v_{1 x}\right]\right) d p\right]=0 \\
& v_{2}=-\mathrm{L}_{p}^{-1} \mathrm{~L}_{s}^{-1}\left[\frac{1}{s} \int_{0}^{p}\left(\mathrm{~L}_{x} \mathrm{~L}_{\mathrm{t}}\left[\left(x v_{1 x}\right)_{x}+\left(x v_{1 x}\right)_{x t}-x^{2} u_{1 x t}\right]\right) d p\right]=0 .
\end{aligned}
$$

In the same manner, we obtain that

$$
u_{3}=0, v_{3}=0
$$

Therefore, the approximate solution is

$$
u(x, t)=u_{0}+u_{1}+\cdots \text { and } v(x, t)=v_{0}+v_{1}+\cdots .
$$

The solution of the system is given by

$$
u(x, t)=x^{2} t \quad \text { and } \quad v(x, t)=x^{2} t .
$$




\section{Nonlinear singular one dimensional pseudo thermo-elasticity coupled system}

The aim of this section is to discuss our method for the nonlinear singular one dimensional pseudo thermo-elasticity coupled system. We consider the general form of nonlinear singular one dimensional pseudo thermo-elasticity coupled system with initial conditions is given below

$$
\begin{aligned}
& \frac{\partial^{2} u}{\partial t^{2}}-\frac{1}{x} \frac{\partial}{\partial x}\left(x \frac{\partial u}{\partial x}\right)-\frac{1}{x} \frac{\partial^{2}}{\partial x \partial t}\left(x \frac{\partial u}{\partial x}\right)+\frac{\partial u}{\partial x} \frac{\partial v}{\partial x}=f(u), \\
& \frac{\partial v}{\partial t}-\frac{1}{x} \frac{\partial}{\partial x}\left(x \frac{\partial v}{\partial x}\right)-\frac{1}{x} \frac{\partial^{2}}{\partial x \partial t}\left(x \frac{\partial v}{\partial x}\right)+\frac{\partial v}{\partial x} \frac{\partial^{2} u}{\partial x \partial t}=g(v),
\end{aligned}
$$

subject to

$$
u(x, 0)=f_{1}(x), \frac{\partial u(x, 0)}{\partial t}=f_{2}(x), \quad v(x, 0)=g_{1}(x),
$$

where $\frac{1}{x}\left(\chi \frac{\partial u}{\partial x}\right)_{\chi}$ and $\frac{1}{\chi}\left(\chi \frac{\partial v}{\partial x}\right)_{\chi}$ are called Bessel's operators, and $f(u)$ and $g(v)$ are nonlinear function. To obtain the solution of nonlinear singular one dimensional pseudo thermo-elasticity coupled system of (3.1), we apply the definition of partial derivatives of the double Laplace transform and single Laplace transform for Eqs. (3.1) and (3.2), respectively and Definition1.1, we get

$$
\begin{aligned}
\frac{d U(p, s)}{d p}= & \frac{d F_{1}(p)}{s d p}+\frac{d F_{1}(p)}{s^{2} d p}-\frac{1}{s^{2}} L_{x} L_{t}\left[\frac{\partial}{\partial x}\left(x \frac{\partial u}{\partial x}\right)\right] \\
& -\frac{1}{s^{2}} L_{x} L_{t}\left[\frac{\partial^{2}}{\partial x \partial t}\left(x \frac{\partial u}{\partial x}\right)-x \frac{\partial u}{\partial x} \frac{\partial v}{\partial x}+x f(u)\right],
\end{aligned}
$$

and

$$
\frac{d V(p, s)}{d p}=\frac{d G_{1}(p)}{s d p}-\frac{1}{s} L_{x} L_{t}\left[\frac{\partial}{\partial x}\left(x \frac{\partial v}{\partial x}\right)\right]-\frac{1}{s} L_{x} L_{t}\left[\frac{\partial^{2}}{\partial x \partial t}\left(x \frac{\partial v}{\partial x}\right)-x \frac{\partial v}{\partial x} \frac{\partial^{2} u}{\partial x \partial t}+x g(v)\right]
$$

applying the integral for both sides of (3.3) and (3.4) from 0 to $p$ with respect to $p$, we have

$$
\begin{aligned}
U(p, s)= & \int_{0}^{p}\left(\frac{d F_{1}(p)}{s d p}+\frac{d F_{1}(p)}{s^{2} d p}\right) d p-\frac{1}{s^{2}} \int_{0}^{p} L_{x} L_{t}\left[\frac{\partial}{\partial x}\left(x \frac{\partial u}{\partial x}\right)\right] d p \\
& -\frac{1}{s^{2}} \int_{0}^{p} L_{x} L_{t}\left[\frac{\partial^{2}}{\partial x \partial t}\left(x \frac{\partial u}{\partial x}\right)-x \frac{\partial u}{\partial x} \frac{\partial v}{\partial x}+x f(u)\right] d p
\end{aligned}
$$

and

$$
\begin{aligned}
V(p, s)= & \int_{0}^{p}\left(\frac{d G_{1}(p)}{s d p}\right) d p-\frac{1}{s} \int_{0}^{p} L_{x} L_{t}\left[\frac{\partial}{\partial x}\left(x \frac{\partial v}{\partial x}\right)\right] d p \\
& -\frac{1}{s} \int_{0}^{p} L_{x} L_{t}\left[\frac{\partial^{2}}{\partial x \partial t}\left(x \frac{\partial v}{\partial x}\right)-x \frac{\partial v}{\partial x} \frac{\partial^{2} u}{\partial x \partial t}+x g(v)\right] d p .
\end{aligned}
$$

The double Laplace a domain decomposition methods (DLADM) defines the solution of the system as $u(x, t)$ and $v(x, t)$ by the infinite series

$$
u(x, t)=\sum_{n=0}^{\infty} u_{n}(x, t), \quad v(x, t)=\sum_{n=0}^{\infty} v_{n}(x, t) .
$$

By applying double inverse Laplace transform for Eqs. (3.5) and (3.6), we have

$$
u(x, t)=L_{p}^{-1} L_{s}^{-1}\left[\int_{0}^{p}\left(\frac{d F_{1}(p)}{s d p}+\frac{d F_{1}(p)}{s^{2} d p}\right) d p\right]
$$




$$
\begin{aligned}
& -\mathrm{L}_{p}^{-1} \mathrm{~L}_{s}^{-1}\left[\frac{1}{s^{2}} \int_{0}^{p} \mathrm{~L}_{x} \mathrm{~L}_{\mathrm{t}}\left[\frac{\partial}{\partial x}\left(\chi \frac{\partial u}{\partial x}\right)\right] d p\right]-\mathrm{L}_{p}^{-1} \mathrm{~L}_{s}^{-1}\left[\frac{1}{s^{2}} \int_{0}^{p} \mathrm{~L}_{x} \mathrm{~L}_{t}\left[\frac{\partial^{2}}{\partial x \partial t}\left(\chi \frac{\partial u}{\partial x}\right)\right] d p\right] \\
& -\mathrm{L}_{p}^{-1} \mathrm{~L}_{s}^{-1}\left[\frac{1}{s^{2}} \int_{0}^{p} \mathrm{~L}_{x} \mathrm{~L}_{t}\left[-\chi \frac{\partial u}{\partial x} \frac{\partial v}{\partial x}+\chi f(u)\right] d p\right]
\end{aligned}
$$

and

$$
\begin{aligned}
v(x, t)= & \mathrm{L}_{p}^{-1} \mathrm{~L}_{s}^{-1}\left[\int_{0}^{p}\left(\frac{d \mathrm{G}_{1}(p)}{s d p}-\frac{1}{s} \mathrm{~L}_{x} \mathrm{~L}_{\mathrm{t}}\left[\frac{\partial}{\partial x}\left(x \frac{\partial v}{\partial x}\right)\right]\right) d p\right] \\
& -\mathrm{L}_{p}^{-1} \mathrm{~L}_{s}^{-1}\left[\frac{1}{s} \int_{0}^{p} \mathrm{~L}_{x} \mathrm{~L}_{\mathrm{t}}\left[\frac{\partial^{2}}{\partial x \partial t}\left(x \frac{\partial v}{\partial x}\right)\right] d p\right] \\
& -\mathrm{L}_{p}^{-1} \mathrm{~L}_{s}^{-1}\left[\frac{1}{s} \int_{0}^{p} \mathrm{~L}_{x} \mathrm{~L}_{\mathrm{t}}\left[-x \frac{\partial v}{\partial x} \frac{\partial^{2} u}{\partial x \partial t}+x g(v)\right] d p\right]
\end{aligned}
$$

where $L_{x} L_{t}$ double Laplace transform with respect to $x, t$ and $L_{p}^{-1} L_{s}^{-1}$ double inverse Laplace transform with respect to $p, s$ and the nonlinear terms $f(u)$ and $g(v)$, such as $u^{2}, u^{3}, u^{4}, \sin u, e^{u}, u u_{x}, u_{x}^{2}$, and $v^{2}$, $v^{3}, v^{4}, \sin v, e^{v}, v v_{x}, v_{x}^{2}$, etc., respectively, can be expressed by an infinite series $A_{n}$ and $B_{n}$ are defined as follows

$$
f(u)=\sum_{i=0}^{\infty} A_{n}\left(u_{0}, u_{1}, u_{2}, \ldots, u_{n}\right), g(v)=\sum_{i=0}^{\infty} B_{n}\left(v_{0}, v_{1}, v_{2}, \ldots, v_{n}\right),
$$

and

$$
A_{n}=\frac{1}{n !}\left(\frac{d^{n}}{d \lambda^{n}}\left[f\left(\sum_{i=0}^{n}\left(\lambda^{i} u_{i}\right)\right)\right]\right)_{\lambda=0}, B_{n}=\frac{1}{n !}\left(\frac{d^{n}}{d \lambda^{n}}\left[g\left(\sum_{i=0}^{n}\left(\lambda^{i} v_{i}\right)\right)\right]\right)_{\lambda=0} .
$$

The first four Adomian polynomials for this term are

$$
\begin{aligned}
& A_{0}=f\left(u_{0}\right), A_{1}=u_{1} f^{\prime}\left(u_{0}\right), \\
& A_{2}=u_{2} f^{\prime}\left(u_{0}\right)+\frac{1}{2 !} u_{1}^{2} f^{\prime \prime}\left(u_{0}\right), \\
& A_{3}=u_{3} f^{\prime}\left(u_{0}\right)+u_{1} u_{2} f^{\prime \prime}\left(u_{0}\right)+\frac{1}{3 !} u_{1}^{3} f^{\prime \prime \prime}\left(u_{0}\right), \\
& A_{4}=u_{4} f^{\prime}\left(u_{0}\right)+\left(\frac{1}{2 !} u_{2}^{2}+u_{1} u_{3}\right) f^{\prime \prime}\left(u_{0}\right)+\frac{1}{2 !} u_{1}^{2} u_{2} f^{(3)}\left(u_{0}\right)+\frac{1}{4 !} u_{1}^{4} u_{2} f^{(4)}\left(u_{0}\right),
\end{aligned}
$$

and

$$
\begin{aligned}
& \mathrm{B}_{0}=\mathrm{g}\left(v_{0}\right), \mathrm{B}_{1}=v_{1} \mathrm{~g}^{\prime}\left(v_{0}\right), \\
& \mathrm{B}_{2}=v_{2} \mathrm{~g}^{\prime}\left(v_{0}\right)+\frac{1}{2 !} v_{1}^{2} \mathrm{~g}^{\prime \prime}\left(v_{0}\right), \\
& \mathrm{B}_{3}=v_{3} \mathrm{~g}^{\prime}\left(v_{0}\right)+v_{1} v_{2} \mathrm{~g}^{\prime \prime}\left(v_{0}\right)+\frac{1}{3 !} v_{1}^{3} \mathrm{~g}^{\prime \prime \prime}\left(v_{0}\right), \\
& \mathrm{B}_{4}=v_{4} \mathrm{~g}^{\prime}\left(v_{0}\right)+\left(\frac{1}{2 !} v_{2}^{2}+v_{1} v_{3}\right) \mathrm{g}^{\prime \prime}\left(v_{0}\right)+\frac{1}{2 !} v_{1}^{2} v_{2} \mathrm{~g}^{(3)}\left(v_{0}\right)+\frac{1}{4 !} v_{1}^{4} v_{2} \mathrm{~g}^{(4)}\left(v_{0}\right),
\end{aligned}
$$

substituting (3.10) and (3.11) into (3.9) gives

$$
f(u)=A_{0}+A_{1}+A_{2}+A_{3}+\cdots, \quad g(v)=B_{0}+B_{1}+B_{2}+B_{3}+\cdots .
$$

We provide the double inverse Laplace transform existing for each terms in the right side of Eqs. (3.7) and (3.8). 


\section{Convergence analysis of the method}

In this section, we will discuss the convergence analysis of the modified double Laplace decomposition methods for the nonlinear singular one dimensional pseudo thermo-elasticity coupled system given by

$$
\left\{\begin{array}{l}
\frac{\partial^{2} u}{\partial t^{2}}-\frac{1}{x} \frac{\partial}{\partial x}\left(x \frac{\partial u}{\partial x}\right)-\frac{1}{x} \frac{\partial^{2}}{\partial x \partial t}\left(x \frac{\partial u}{\partial x}\right)+\frac{\partial u}{\partial x} \frac{\partial v}{\partial x}=f(u) \\
\frac{\partial v}{\partial t}-\frac{1}{x} \frac{\partial}{\partial x}\left(x \frac{\partial v}{\partial x}\right)-\frac{1}{x} \frac{\partial^{2}}{\partial x \partial t}\left(x \frac{\partial v}{\partial x}\right)+\frac{\partial v}{\partial x} \frac{\partial^{2} u}{\partial x \partial t}=g(v) .
\end{array}\right.
$$

We propose to extend this idea given in $[18,19]$. First of all, let us consider the Hilbert space $\mathrm{H}=$ $\mathrm{L}_{\mu}^{2}((\mathrm{a}, \mathrm{b}) \times[0, \mathrm{~T}])$, defined by the set of applications

$$
\left\{\begin{array}{l}
(u, v):(a, b) \times[0, T], \text { with } \\
\mathrm{L}_{p}^{-1} \mathrm{~L}_{s}^{-1}\left[\frac{1}{s^{2}} \int_{0}^{p} \mathrm{~L}_{x} \mathrm{~L}_{t}[u(x, t)](p, s) d p\right](x, t)<\infty
\end{array}\right.
$$

where

$$
u:(a, b) \times[0, T] \rightarrow \mathbb{R}, \text { with }\|u\|_{H}^{2}=\int_{Q} x u^{2}(x, t) d x d t
$$

the scalar product

$$
(u, v)_{L_{\mu}^{2}(Q)}=\int_{Q} x u(x, t) v(x, t) d x d t,
$$

and $Q=(a, b) \times[0, T]$. For more details see [7]. We can rewrite (4.1) in the following form

$$
\left\{\begin{array}{l}
\mathrm{L}(\mathrm{u})=x \frac{\partial^{2} u}{\partial \mathrm{t}^{2}}=\frac{\partial u}{\partial x}+x \frac{\partial^{2} u}{\partial x^{2}}+\frac{\partial^{2} u}{\partial x \partial t}+x \frac{\partial^{3} u}{\partial x^{2} \partial t}-x \frac{\partial u}{\partial x} \frac{\partial v}{\partial x}+x f(u) \\
\mathrm{L}(v)=x \frac{\partial v}{\partial t}=\frac{\partial v}{\partial x}+x \frac{\partial^{2} v}{\partial x^{2}}+\frac{\partial^{2} v}{\partial x \partial t}+x \frac{\partial^{3} v}{\partial x^{2} \partial t}-x \frac{\partial v}{\partial x} \frac{\partial^{2} u}{\partial x \partial t}+x g(v)
\end{array}\right.
$$

The modified double Laplace decomposition methods is convergence if the following two hypotheses are satisfied:

(H1) $(\mathrm{L}(\mathrm{u})-\mathrm{L}(w), \mathrm{u}-w) \geqslant \mathrm{k}\|\mathrm{u}-w\|^{2}$ and $(\mathrm{L}(v)-\mathrm{L}(w), v-w) \geqslant k\|v-w\|^{2} ; \mathrm{k}>0, \forall \mathrm{u}, v, w \in \mathrm{H}$;

(H2) whatever may be $M>0$, there exists a constant $C(M)>0$ such that for $u, w \in H$ with $\|u\| \leqslant$ $M,\|v\| \leqslant M,\|w\| \leqslant M$, and $\left\|\frac{\partial^{2} u}{\partial x \partial t}\right\| \leqslant M_{2},\left\|\frac{\partial v}{\partial x}\right\| \leqslant M_{1}$, we have

$$
\left(\mathrm{L}(\mathrm{u})-\mathrm{L}(w), z_{1}\right) \leqslant \mathrm{C}(\mathrm{M})\left\|\mathrm{u}-z_{1}\right\|\|w\|, \text { and }\left(\mathrm{L}(v)-\mathrm{L}\left(z_{2}\right), w\right) \leqslant \mathrm{C}(\mathrm{M})\left\|v-z_{2}\right\|\|w\|
$$

for every $z_{1}, z_{2} \in \mathrm{H}$.

In the next Theorem we follow $[11,13,14]$.

Theorem 4.1 (Sufficient condition of convergence). The Modified double Laplace decomposition method applied to the nonlinear singular one dimensional pseudo thermo-elasticity system (4.2) without initial and boundary conditions, converges towards a particular solution.

Proof. First, we verify the convergence hypothesis $(\mathrm{H} 1)$ for the operators $\mathrm{L}(\mathrm{u}), \mathrm{L}(v)$ of $(4.2)$. We use the definition of our operator $L$, and then we have

$$
\begin{aligned}
L(u)-L(w)= & \left(\frac{\partial u}{\partial x}-\frac{\partial w}{\partial x}\right)+\left(x \frac{\partial^{2} u}{\partial x^{2}}-x \frac{\partial^{2} w}{\partial x^{2}}\right)\left(\frac{\partial^{2} u}{\partial x \partial t}-\frac{\partial^{2} w}{\partial x \partial t}\right)+\left(x \frac{\partial^{3} u}{\partial x^{2} \partial t}-x \frac{\partial^{3} w}{\partial x^{2} \partial t}\right) \\
& -\left(x \frac{\partial v}{\partial x} \frac{\partial u}{\partial x}-x \frac{\partial v}{\partial x} \frac{\partial w}{\partial x}\right)+x(f(u)-f(w)) \\
= & \frac{\partial}{\partial x}(u-w)+x \frac{\partial^{2}}{\partial x^{2}}(u-w)+\frac{\partial^{2}}{\partial x \partial t}(u-w) \\
& +x \frac{\partial^{3}}{\partial x^{2} \partial t}(u-w)-x \frac{\partial v}{\partial x} \frac{\partial}{\partial x}(u-w)+x(f(u)-f(w)),
\end{aligned}
$$


and

$$
\begin{aligned}
L(v)-L(w)= & \left(\frac{\partial v}{\partial x}-\frac{\partial w}{\partial x}\right)+\left(x \frac{\partial^{2} v}{\partial x^{2}}-x \frac{\partial^{2} w}{\partial x^{2}}\right)\left(\frac{\partial^{2} v}{\partial x \partial t}-\frac{\partial^{2} w}{\partial x \partial t}\right)+\left(x \frac{\partial^{3} v}{\partial x^{2} \partial t}-x \frac{\partial^{3} w}{\partial x^{2} \partial t}\right) \\
& -\left(x \frac{\partial^{2} u}{\partial x \partial t} \frac{\partial v}{\partial x}-x \frac{\partial^{2} u}{\partial x \partial t} \frac{\partial w}{\partial x}\right)+x(g(v)-g(w)) \\
= & \frac{\partial}{\partial x}(v-w)+x \frac{\partial^{2}}{\partial x^{2}}(v-w)+\frac{\partial^{2}}{\partial x \partial t}(v-w) \\
& +x \frac{\partial^{3}}{\partial x^{2} \partial t}(v-w)-x \frac{\partial^{2} u}{\partial x \partial t} \frac{\partial}{\partial x}(v-w)+x(g(v)-g(w)),
\end{aligned}
$$

therefore,

$$
\begin{aligned}
(L(u)-L(w), u-w)= & \left(\frac{\partial}{\partial x}(u-w), u-w\right)+\left(x \frac{\partial^{2}}{\partial x^{2}}(u-w), u-w\right) \\
& +\left(\frac{\partial^{2}}{\partial x \partial t}(u-w), u-w\right)+\left(x \frac{\partial^{3}}{\partial x^{2} \partial t}(u-w), u-w\right) \\
& -\left(x \frac{\partial v}{\partial x} \frac{\partial}{\partial x}(u-w), u-w\right)+(x(f(u)-f(w)), u-w),
\end{aligned}
$$

and

$$
\begin{aligned}
(\mathrm{L}(v)-\mathrm{L}(w), v-w)= & \left(\frac{\partial}{\partial x}(v-w), v-w\right)+\left(x \frac{\partial^{2}}{\partial x^{2}}(v-w), v-w\right) \\
& +\left(\frac{\partial^{2}}{\partial x \partial t}(v-w), v-w\right)+\left(x \frac{\partial^{3}}{\partial x^{2} \partial t}(v-w), v-w\right) \\
& -\left(x \frac{\partial^{2} u}{\partial x \partial t} \frac{\partial}{\partial x}(v-w), v-w\right)+(x(g(v)-g(w)), v-w)
\end{aligned}
$$

According to the properties of the differential operators $\frac{\partial}{\partial x}$ and $\frac{\partial^{2}}{\partial x \partial t}$ in $H$, then there exists constants $\alpha, \beta, \delta, \theta, \phi>0$ such that

$$
\left(\frac{\partial}{\partial x}(u-w), u-w\right) \geqslant \alpha\|u-w\|^{2},
$$

and

$$
\begin{aligned}
-\left(x \frac{\partial^{2}}{\partial x^{2}}(u-w), u-w\right) & \leqslant|x|\left\|\frac{\partial^{2}}{\partial x^{2}}(u-w)\right\|\|u-w\| \\
& \leqslant b \beta\|u-w\|^{2} \\
& \Leftrightarrow \\
\left(x \frac{\partial^{2}}{\partial x^{2}}(u-w), u-w\right) & \geqslant-b \beta\|u-w\|^{2}, \\
\left(\frac{\partial^{2}}{\partial x \partial t}(u-w), u-w\right) & \geqslant \theta\|u-w\|^{2},
\end{aligned}
$$

and

$$
\begin{aligned}
-\left(x \frac{\partial^{3}}{\partial x^{2} \partial t}(u-w), u-w\right) & \leqslant|x|\left\|\frac{\partial^{3}}{\partial x^{2} \partial t}(u-w)\right\|\|u-w\| \\
& \leqslant b \phi\|u-w\|^{2} \\
& \Leftrightarrow \\
\left(x \frac{\partial^{2}}{\partial x^{2}}(u-w), u-w\right) & \geqslant-b \phi\|u-w\|^{2}
\end{aligned}
$$


and according to the Schwartz inequality, we get

$$
\left(x \frac{\partial v}{\partial x} \frac{\partial}{\partial x}(u-w), u-w\right) \leqslant|x|\left\|\frac{\partial v}{\partial x}\right\|\left\|\frac{\partial}{\partial x}(u-w)\right\|\|u-w\| \leqslant M_{1} b \alpha\|u-w\|^{2},
$$

hence,

$$
-\left(x \frac{\partial v}{\partial x} \frac{\partial}{\partial x}(u-w), u-w\right) \geqslant-M_{1} b \alpha\|u-w\|^{2} .
$$

Again by using Cauchy Schwarz inequality, where $f$ is Lipschitzian function, we have

$$
\begin{aligned}
(-x(f(u)-f(w)), u-w) & \leqslant|x|\|f(u)-f(w)\|\|u-w\| \\
& \leqslant b\|f(u)-f(w)\|\|u-w\| \\
& \leqslant b \delta\|u-w\|^{2} \\
& \Leftrightarrow \\
(x(f(u)-f(w)), u-w) & \geqslant-b \delta\|u-w\|^{2} .
\end{aligned}
$$

Substituting Eqs. (4.5), (4.6), (4.7), (4.9), (4.10), and (4.8) into (4.3) yields

$$
\begin{aligned}
& (\mathrm{L}(\mathrm{u})-\mathrm{L}(w), u-w) \geqslant\left(\alpha-\mathrm{b} \beta+\theta-\mathrm{b} \phi-\mathrm{M}_{1} \mathrm{~b} \alpha-\mathrm{b} \delta\right)\|\mathrm{u}-w\|^{2}, \\
& (\mathrm{~L}(\mathrm{u})-\mathrm{L}(w), u-w) \geqslant k_{1}\|\mathrm{u}-w\|^{2},
\end{aligned}
$$

where $k_{1}=\alpha-b \beta+\theta-b \phi-M_{1} b \alpha-b \delta>0$.

In the same way, for (4.4) there exists constants $\zeta, \eta, \lambda, \rho, \sigma>0$ such that

$$
\begin{aligned}
& (\mathrm{L}(v)-\mathrm{L}(w), v-w) \geqslant\left(\zeta-\mathrm{b} \eta+\sigma-\mathrm{b} \rho-\mathrm{M}_{2} \zeta \mathrm{b}-\mathrm{b} \lambda\right)\|v-w\|^{2}, \\
& (\mathrm{~L}(v)-\mathrm{L}(w), v-w) \geqslant k_{2}\|v-w\|^{2},
\end{aligned}
$$

where $k_{2}=\zeta-b \eta+\sigma-b \rho-M_{2} \zeta b-b \lambda>0$. Hence hypothesis (H1) holds. Now, we verify the convergence hypotheses $(\mathrm{H} 2)$ for the operators $L(u)$ and $L(v)$. For every $M>0$, there exists a constant $C(M)$ $>0$ such that for all $u, v, w \in H$ with $\|u\| \leqslant M,\|v\| \leqslant M,\left\|\frac{\partial^{2} u}{\partial x \partial t}\right\| \leqslant M_{2}$, and $\left\|\frac{\partial v}{\partial x}\right\| \leqslant M_{1}$, we have

$$
\left(\mathrm{L}(\mathrm{u})-\mathrm{L}(\mathfrak{w}), z_{1}\right) \leqslant \mathrm{C}(\mathrm{M})\|\mathrm{u}-\mathfrak{w}\|\left\|z_{1}\right\|
$$

for every $z_{1}, z_{2} \in \mathrm{H}$.

For that we have,

$$
\begin{aligned}
\left(\mathrm{L}(\mathrm{u})-\mathrm{L}(w), z_{1}\right)= & \left(\frac{\partial}{\partial x}(u-w), z_{1}\right)+\left(x \frac{\partial^{2}}{\partial x^{2}}(u-w), z_{1}\right) \\
& +\left(\frac{\partial^{2}}{\partial x \partial t}(u-w), z_{1}\right)+\left(x \frac{\partial^{3}}{\partial x^{2} \partial t}(u-w), z_{1}\right) \\
& -\left(x \frac{\partial v}{\partial x} \frac{\partial}{\partial x}(u-w), z_{1}\right)+\left(x(f(u)-f(w)), z_{1}\right) .
\end{aligned}
$$

Similarly, by using the Cauchy Schwartz inequality and the fact that $u$ and $w$ are bounded, we obtain the following:

$$
\begin{gathered}
\left(\frac{\partial}{\partial x}(u-w), z_{1}\right) \leqslant \alpha_{1}\|u-w\|\left\|z_{1}\right\|, \\
\left(x \frac{\partial^{2}}{\partial x^{2}}(u-w), z_{1}\right) \leqslant b \beta_{1}\|u-w\|\left\|z_{1}\right\|,
\end{gathered}
$$




$$
\begin{aligned}
\left(\frac{\partial^{2}}{\partial x \partial t}(u-w), z_{1}\right) & \leqslant \alpha_{2}\|u-w\|\left\|z_{1}\right\| \\
\left(x \frac{\partial^{3}}{\partial x^{2} \partial t}(u-w), z_{1}\right) & \leqslant b \alpha_{3}\|u-w\|\left\|z_{1}\right\| \\
-\left(x \frac{\partial v}{\partial x} \frac{\partial}{\partial x}(u-w), z_{1}\right) & \leqslant \alpha_{1}|x|\left\|\frac{\partial v}{\partial x}\right\|\|u-w\|\left\|z_{1}\right\| \leqslant b \alpha_{1} M_{1}\|u-w\|\left\|z_{1}\right\|,
\end{aligned}
$$

and

$$
\left(x(f(u)-f(w)), z_{1}\right) \leqslant b \sigma_{1}\|u-w\|\left\|z_{1}\right\|
$$

where the constants $\alpha_{1}, \beta_{1}, \alpha_{2}, \alpha_{3}, \sigma_{1}>0$, we have

$$
\left(\mathrm{L}(\mathrm{u})-\mathrm{L}(w), z_{1}\right) \leqslant\left(\alpha_{1}+\mathrm{b} \beta_{1}+\alpha_{2}+\mathrm{b} \alpha_{3}+\mathrm{b} \alpha_{2} \mathrm{M}_{1}+\mathrm{b} \sigma_{1}\right)\|\mathrm{u}-w\|\left\|z_{1}\right\|=\mathrm{C}_{1}(\mathrm{M})\|\mathrm{u}-w\|\left\|z_{1}\right\|,
$$

where

$$
C_{1}(M)=\alpha_{1}+b \beta_{1}+\alpha_{2}+b \alpha_{3}+b \alpha_{2} M_{1}+b \sigma_{1}
$$

and

$$
\begin{aligned}
\left(\mathrm{L}(v)-\mathrm{L}(w), z_{2}\right)= & \left(\frac{\partial}{\partial x}(v-w), z_{2}\right)+\left(x \frac{\partial^{2}}{\partial x^{2}}(v-w), z_{2}\right)+\left(\frac{\partial^{2}}{\partial x \partial t}(v-w), z_{2}\right) \\
& +\left(x \frac{\partial^{3}}{\partial x^{2} \partial t}(v-w), z_{2}\right)-\left(x \frac{\partial^{2} u}{\partial x \partial t} \frac{\partial}{\partial x}(v-w), z_{2}\right)+\left(x(g(v)-f(w)), z_{2}\right)
\end{aligned}
$$

In the same way, we obtain,

$$
\left(\mathrm{L}(v)-\mathrm{L}(w), z_{2}\right) \leqslant\left(\zeta_{1}+\mathrm{b} \eta_{1}+\zeta_{2}+\mathrm{b} \zeta_{3}+\mathrm{b} \zeta_{2} \mathrm{M}_{2}+\mathrm{b} \rho_{1}\right)\|v-w\|\left\|z_{2}\right\|=\mathrm{C}_{2}(\mathrm{M})\|v-w\|\left\|z_{2}\right\|,
$$

where

$$
\mathrm{C}_{2}(\mathrm{M})=\zeta_{1}+\mathrm{b} \eta_{1}+\zeta_{2}+\mathrm{b} \zeta_{3}+\mathrm{b} \zeta_{2} \mathrm{M}_{2}+\mathrm{b} \rho_{1}
$$

and $\zeta_{1}, \eta_{1}, \zeta_{2}, \zeta_{3}, \rho_{1}>0$, therefore (H2) holds.

Conclusion 4.2. In this paper, first a double Laplace transform algorithm which is based on the Adomian decomposition method is used for solving the linear and nonlinear singular one dimensional pseudo thermo-elasticity coupled system. Second, we presented a convergence proof of the (DLADM) applied to the linear and nonlinear singular one dimensional pseudo thermo-elasticity coupled system.

\section{Acknowledgment}

The authors would like to extend their sincere appreciation to the Deanship of Scientific Research at King Saud University for its funding this Research group No (RG-1435-043).

\section{References}

[1] K. Abbaoui, Y. Cherruault, Convergence of Adomian's Method Applied to Differential Equations, Comput. Math. Model., 28 (1994), 103-109. 1

[2] K. Abbaoui, Y. Cherruault, Convergence of Adomian's Method Applied to Nonlinear Equations, Math. Comput. Modelling., 20 (1994), 69-73.

[3] K. Abbaoui, Y. Cherruault, V. Seng, Practical Formulae for the Calculus of Multivariable Adomian Polynomials, Math. Comput. Modelling., 22 (1995), 89-93. 1

[4] F. M. Allan, K. Al-khaled, An approximation of the analytic solution of the shock wave equation, J. Comput. Appl. Math., 192 (2006), 301-309. 1

[5] A. Atangana, Convergence and stability analysis of a novel iteration method for fractional biological population equation, Neural Comput \& Applic., 25 (2014), 1021-1030. 1 
[6] A. Atangana, On the new fractional derivative and application to nonlinear Fisher's reaction-diffusion equation, Appl. Math. Comput., 273 (2016), 948-956. 1

[7] A. Atangana, S. C. Oukouomi Noutchie, On Multi-Laplace Transform for Solving Nonlinear Partial Differential Equations with Mixed Derivatives, Math. Probl. Eng., 2014 (2014), 9 pages. 1, 4

[8] E. Babolian, J. Biazar, On the order of convergence of Adomian method, Appl. Math. Comput., 130 (2002), 383-387. 1

[9] S. M. El-Sayed, D. Kaya, On the numerical solution of the system of two-dimensional Burgers' equations by the decomposition method, Appl. Math. Comput., 158 (2004), 101-109. 1

[10] H. Eltayeb, A. Kilıçman, A Note on Solutions of Wave, Laplace's and Heat Equations with Convolution Terms by Using Double Laplace Transform, Appl. Math. Lett., 21 (2008), 1324-1329. 1

[11] I. Hashim, M. S. M. Noorani, M. R. Said Al-Hadidi, Solving the generalized Burgers-Huxley equation using the Adomian decomposition method, Math. Comput. Modelling., 43 (2006), 1404-1411. 1, 4

[12] J.-H. He, Homotopy perturbation method for solving boundary value problems, Phys. Lett. A, 350 (2006), 87-88. 1

[13] D. Kaya, I. E. Inan, A convergence analysis of the ADM and an application, Appl. Math. Comput., 161 (2005), 10151025. 4

[14] D. Kaya, I. E. Inan, A numerical application of the decomposition method for the combined KdV-MKdV equation, Appl. Math. Comput., 168 (2005), 915-926. 4

[15] A. Kılıçman, H. Eltayeb, A note on defining singular integral as distribution and partial differential equation with convolution term, Math. Comput. Modelling., 49 (2009), 327-336. 1

[16] A. Kılıçman, H. E. Gadain, On the applications of Laplace and Sumudu transforms, J. Franklin Inst., 347 (2010), 848 862. 1

[17] H. Lord, Y. Shulman, A generalized dynamical theory of thermo-elasticity, J. Mech. Phys. Solids., 15 (1967), 299-309. 1

[18] T. Mavoungou, Y. Cherruault, Convergence of Adomian's method and applications to nonlinear partial differential equations, Kybernetes, 21 (1992), 13-25. 4

[19] T. Mavoungou, Y. Cherruault, Numerical study of Fisher's equation by Adomian's method, Math. Comput. Modelling., 19 (1994), 89-95. 4

[20] S. Mesloub, F. Mesloub, On a coupled nonlinear singular thermoelastic system, Nonlinear Anal., 73 (2010), $3195-3208$. 1

[21] S. S. Ray, A numerical solution of the coupled sine-gordon equation using the modified decomposition method, Appl. Math. Comput., 175 (2006), 1046-1054. 1

[22] N. H. Sweilam, Harmonic wave generation in nonlinear thermoelasticity by variational iteration method and adomian's method, J. Comput. Appl. Math., 207 (2007), 64-72. 1

[23] N. H. Sweilam, M. M. Khader, Variational iteration method for one dimensional nonlinear thermoelasticity, Chaos Solitons Fractals, 32 (2007), 145-149. 1

[24] A.-M. Wazwaz, Partial Differential Equations and Solitary Waves Theory, Springer, Berlin, (2009). 2 